\title{
ARITHMETICAL CONSEQUENCES OF A TRIGONOMETRIC IDENTITY
}

BY E. T. BELL

1. Identities. The formulas for the product of $s$ sines, $t$ cosines, or $s$ sines and $t$ cosines, give immediate elementary proofs concerning representations of numbers in certain forms of degrees $s, t, s+t$. Here we consider $s$ sines, where $s>1$. Let us write

$$
\psi_{n}\left(x_{1}, \cdots, x_{s}\right) \equiv \sum e_{2} \cdots e_{s}\left(x_{1}+e_{2} x_{2}+\cdots+e_{s} x_{s}\right)^{n},
$$

where $\sum$ refers to the $2^{s-1}$ possible sets $\left(e_{2}, \cdots, e_{s}\right), e_{j}= \pm 1$, $(j=2, \cdots, s)$. Then, for $t>0$,

$$
\begin{aligned}
2^{2 t-1}(-1)^{t} \prod_{j=1}^{2 t} \sin x_{j} \theta & =\cos \psi\left(x_{1}, \cdots, x_{2 t}\right) \theta, \\
2^{2 t}(-1)^{t} \prod_{j=1}^{2 t+1} \sin x_{j} \theta & =\sin \psi\left(x_{1}, \cdots, x_{2 t+1}\right) \theta,
\end{aligned}
$$

in which, after expansion of the (symbolic) cos, sin on the right, $\psi^{n}()$ is to be replaced by $\psi_{n}($ ). Such expansions were discussed in detail in a previous paper.*

The results of equating coefficients of $\theta^{2 t}, \theta^{2 t+1}$ in these can be combined at once in to the single identity

$$
s ! 2^{s-1} x_{1} \cdots x_{s}=\psi_{s}\left(x_{1}, \cdots, x_{s}\right) .
$$

For $s=3$, (1) is due to Gauss, for $s>1$, to Tardy, who proved a slightly different form of (1) otherwise. $\dagger$ In the same way we see that

$$
\psi_{n}\left(x_{1}, \cdots, x_{s}\right)=0, \quad n<s .
$$

In (1) let $x_{1}=x, x_{j}=1(j>1)$. If precisely $p$ of $e_{2}, \cdots, e_{s}$ are $+1, e_{2}+\cdots+e_{s}=2 p+1-s, e_{2} \cdots e_{s}=(-1)^{s-1-p}$. The $p$ can be chosen in ${ }_{s-1} C_{p}$ ways. Hence we have

$$
(-1)^{s-1} s ! 2^{s-1} x=\sum_{p=0}^{s-1}(-1)^{p}{ }_{s-1} C_{p}(x+2 p+1-s)^{s} .
$$

* Transactions of this Society, vol. 38(1926), pp. 129-148.

† Annali di Scienze Matematische e Fisiche, vol. 2(1851), pp. 287-291. 
In (1) let $x_{1}=x_{2}=x, x_{j}=1(j>2)$. Separating the terms according as $e=1$ or -1 , and proceeding as before, we get

(4) $(-1)^{s} s ! 2^{s-1} x^{2}=\sum_{p=0}^{s-2}(-1)^{p}{ }_{s-2} C_{p}\left[(2 x+2 p+2-s)^{s}\right.$

$$
\left.-(2 p+2-s)^{s}\right] \text {. }
$$

Similarly,

(5) $(-1)^{s} s ! 2^{s-1} x^{3}=\sum_{p=0}^{s-3}(-1)^{p}{ }_{s-3} C_{p}\left[2(x+2 p+3-s)^{s}\right.$

$$
\left.-(-x+2 p+3-s)^{s}-(3 x+2 p+3-s)^{s}\right] .
$$

The general formula of the same sort for $x^{n}, n>3$, is easily obtained by the methods of the first paper cited, on using the expansions for powers of sines in terms of multiple angles.

From (3)-(5), by differentiation with respect to $x$, we obtain

$$
\begin{aligned}
& \sum_{p=0}^{s-1}(-1)^{p}{ }_{s-1} C_{p}(x+2 p+1-s)^{s-j}=0, \quad(j>1) ; \\
& \sum_{p=0}^{s-1}(-1)^{p}{ }_{s-2} C_{p}(2 x+2 p+2-s)^{s-j}=0, \quad(j>2) ; \\
& \sum_{p=0}^{s-1}(-1)^{p}{ }_{s-3} C_{p}\left[2(x+2 p+3-s)^{s-j}\right. \\
& \left.-(-x+2 p+3-s)^{s-j}-(3 x+2 p+3-s)^{s-j}\right]=0, \\
& (j>3) \text {, }
\end{aligned}
$$

valid for all $x$.

2. Theorems on Representations. (I) Let $\alpha$ be any integer in an algebraic number field $K$ of (arbitrary) degree $k$. Then

$$
\begin{aligned}
& s ! 2^{s-1} \alpha=e_{1} \alpha_{1}{ }^{s}+\cdots+e_{n} \alpha_{n}^{s}, \quad\left(n \leqq 2^{s-1}\right), \\
& (-1)^{s-1} s ! 2^{s-1} \alpha=\sum_{p=0}^{s-1}(-1)^{p}{ }_{s-1} C_{p} \beta_{p}^{s},
\end{aligned}
$$

where the $\alpha$ 's, $\beta$ 's are integers in $K$. These follow at once from (1), (3). In the first we may take $\alpha=\gamma_{1} \cdots \gamma_{s}, x_{j}=\gamma_{j}$, where the $\gamma$ 's are integers in $K$. If $k=1$, the $\alpha$ 's are rational integers, and if $t$ is the least integer such that $\tau$ is represented in the form 
$e_{1} \alpha_{1}^{8}+\cdots+e_{t} \alpha_{t}^{s}$, all $\alpha \equiv \tau \bmod s ! 2^{n-1}$ are represented in (9) with $n \leqq 2^{s-1}+t$.

(II) Since every (rational) integer $m$ is a sum of four (rational integer) squares, it follows from (5) that

$$
(-1)^{s} s ! 2^{s-1} m+4 \sum_{p=0}^{s-2}{ }_{s-2} C_{p}(2 p+2-s)^{s}
$$

is a sum of 4 values of

$$
\sum_{p=0}^{s-2}(-1)^{p}{ }_{s-2} C_{p} m_{p}{ }^{s},
$$

where the $m$ 's are integers.

Similarly, from (5), every $(-1) s ! 2^{s-1} m$ is a sum of 9 values of

$$
\sum_{p=0}^{s-3}(-1)^{p}{ }_{s-3} C_{p}\left(2 m_{p}^{s}-n_{p}^{s}-r_{p}^{s}\right),
$$

where $m, n, p$ are integers. Theorems of this sort can be continued indefinitely.

3. A Rational Canonical. Let $P \equiv P\left(y_{1}, \cdots, y_{n}\right)$ be a polynomial (not necessarily homogeneous) of degree $s$ in $y_{1}, \cdots, y_{n}$, containing precisely $p$ terms. Then immediately from (1),

$$
s ! 2^{s-1} P=\sum_{j=1}^{h} A_{j} Y_{j}{ }^{s}, \quad h \leqq 2^{s-1} p,
$$

where the $A$ 's are linear in the coefficients of $P$, and the $Y$ 's are linear in $y_{1}, \cdots, y_{n}$, both with rational integer coefficients.

4. Equal Sums of Like Powers. From (2) we have an infinity of solutions of

$$
\begin{array}{r}
a_{1}^{n}+\cdots+a_{t}^{n}=b_{1}^{n}+\cdots+b_{t}^{n}, \quad t=2^{s-2}, \\
(n=0,1, \cdots, s-1),
\end{array}
$$

in integers $a, b$. Similarly from (6)-(8). Thus from (6), for example,

$$
\begin{aligned}
\sum_{p=0}^{s-1} & (-1)^{p}{ }_{s-1} C_{p}(x+2 p+1-s)^{s-j} \\
& =\sum_{p=0}^{s-1}(-1)^{p}{ }_{s-1} C_{p}(y+2 p+1-s)^{s-1}
\end{aligned}
$$


for $0<j \leqq s$, and $x, y$ arbitrary. Hence, for the same $j$,

$$
\begin{aligned}
& \sum_{p=0}^{2 p \leqq s-1}{ }_{s-1} C_{2 p}(x+4 p+1-s)^{s-j} \\
& \quad+\sum_{p=0}^{2 p \leqq s-2} s-1 C_{2 p+1}(y+4 p+3-s)^{s-} \\
& =\sum_{p=0}^{2 p \leqq s-2}{ }_{s-1} C_{2 p+1}(x+4 p+3-s)^{s-1} \\
& \quad+\sum_{p=0}^{2 p \leqq s-1}{ }_{s-1} C_{2 p}(y+4 p+1-s)^{s-j}
\end{aligned}
$$

One term on each side can be made to vanish by suitably choosing $x, y$. Thus we have a solution of

$$
\begin{aligned}
& a_{1}{ }^{n}+\cdots+a_{u}{ }^{n}=b_{1}{ }^{n}+\cdots+b_{u}{ }^{n}, u=2^{s-2}-1, \\
&(n=0, \cdots, s-1) .
\end{aligned}
$$

Similar devices give corresponding results for any $u \leqq 2^{s-2}$, and solutions containing parameters can also be given.

California Institute of Technology

\section{A CORRECTION IN THE LIST OF PRIMES}

BY D. N. LEHMER

My attention has been called by Andr. W. Mund of Basel, Switzerland, to a second error in the List of Primes published for me by the Carnegie Institution of Washington in 1914. The number 854651 listed in those tables is not a prime and should read 854647 . The error is due to an error hitherto undiscovered in Burckhardt's Tables of Divisors, in which an entry of 7 has slipped up one line. The error does not occur in my Factor Tables, and was introduced into the list from Burckhardt's tables, which were more convenient to read than the Carnegie tables.

All users of these tables should insert this correction. It is the second error to be discovered so far. (See this Bulletin, vol. 38 (1932), p. 902.)

The University of California 